Mathematical Modelling and Analysis

Volume 20 Number 6, November 2015, 768-781

http://dx.doi.org/10.3846/13926292.2015.1112856

(c) Vilnius Gediminas Technical University, 2015
Publisher: Taylor\&Francis and VGTU

http://www.tandfonline.com/TMMA

ISSN: $1392-6292$

eISSN: $1648-3510$

\title{
Dunkl-Poisson Equation and Related Equations in Superspace
}

\author{
Hong Fen Yuan $^{a}$ and Valery V. Karachik ${ }^{b}$ \\ ${ }^{a}$ Hebei University of Engineering \\ 056038 Handan, China \\ ${ }^{b}$ South Ural State University \\ 454080 Chelyabinsk, Russia \\ E-mail(corresp.): yuanhongfen1980@126.com \\ E-mail: Karachik@susu.ru
}

Received March 13, 2015; revised September 30, 2015; published online November 15, 2015

\begin{abstract}
In this paper, we investigate the Almansi expansion for solutions of Dunkl-polyharmonic equations by the 0-normalized system for the Dunkl-Laplace operator in superspace. Moreover, applying the 0-normalized system, we construct solutions to the Dunkl-Helmholtz equation, the Dunkl-Poisson equation, and the inhomogeneous Dunkl-polyharmonic equation in superspace.
\end{abstract}

Keywords: normalized system, super Dunkl-Laplace operator, Dunkl-Helmholtz equation, Dunkl-Poisson equation, inhomogeneous Dunkl-polyharmonic equation.

AMS Subject Classification: 30G35; 58C50; 33C52; 35J05.

\section{Introduction}

Dunkl operators (differential-difference operators), introduced by Dunkl [9], are invariant under a finite reflection group $G$ and are also pairwise commuting. One of the interesting aspects of these operators is that they allow for the construction of a Dunkl Laplace operator, which is a combination of the classical Laplace operator in $\mathbb{R}^{m}$ with some difference terms, such that the resulting operator is only invariant under $\mathrm{G}$ and not under the whole orthogonal group. Moreover, these operators not only provide a useful tool in the study of special functions with root systems [10], but they are also closely related to affine Hecke algebras [26] and integrable system of Calogero-Moser-Sutherland type [13].

One of the main aims of Clifford analysis is to study the function-theoretical properties of the null-solutions of the Dirac operator which is invariant under rotations but not under reflections [7]. A Dunkl version of the Dirac operator in Clifford analysis introduced by Cerejeiras et al, is invariant under reflection groups and also factorizes the Dunkl-Laplacian [8]. Then, they obtained a 
Stokes theorem, a Borel-Pompeiu formula and a Cauchy integral formula for the Dirac operator, and investigated a Fueter's theorem and Fischer decompositions in Dunkl Clifford analysis(see [3, 11, 12]). More recently, Sommen, DeBie and others studied Clifford analysis in superspace $\mathbb{R}^{m \mid 2 n}$ (see $[4,5]$ ). Superspaces are spaces equipped with both a set of commuting variables and a set of anticommuting variables in order to describe the properties of bosons and fermions in Quantum Mechanics. In 2010, Ren gave the Fischer decomposition on the space of spinor valued polynomials in the superspace for the super DunklDirac operator with the bosonic Dunkl-Dirac operator (i.e., the Dunkl-Dirac operator in $\mathbb{R}^{m}$ ) and the fermionic Dirac operator(see [24]). Based on the above-mentioned results, we investigated the Almansi type expansion for super Dunkl-Laplace operators.

In 1899, the Almansi expansion for polyharmonic functions was established [1]. Indeed the expansion builds the relation between harmonic functions and polyharmonic functions, which plays a central role in the theory of polyharmonic functions. The result in the case of harmonic analysis, complex analysis, Clifford analysis, and Clifford analysis in superspace have been well developed in $[2,19,21,23,25,28]$. In the present paper, we study Almansi type expansions for solutions of Dunkl-polyharmonic equations in superspace by normalized systems.

Normalized systems of functions were advocated by Bondarenko [6]. Afterwards, Karachik constructed 0-normalized system of functions with respect to a Laplace operator and applied the system to an expansion of Almansi type for polyharmonic functions in $\mathbb{R}^{m}$ (see $[14,15,16]$ ). Then, normalized systems for wave operators, Dunkl operators, super Dirac operators are obtained (see $[20,22,27])$. In particular, applying normalized systems and Almansi expansions, Karachik studied solutions of some partial equations and some boundary value problems for Poisson's Equation (see $[17,18]$ ). But as far as we know, up to now there is no hint on normalized systems in Dunkl superspace. In this paper, we try to fill part of this gap by studying solutions to Dunkl-Helmholtz equations, Dunkl-Poisson equations, inhomogeneous Dunkl-polyharmonic equations in superspace using normalized systems.

\section{Preliminaries}

\subsection{Dunkl-Clifford analysis in $\mathbb{R}^{m}$}

Denote by $\langle.,$.$\rangle the standard Euclidean scalar product in \mathbb{R}^{m}$ and by $|x|=$ $\langle x, x\rangle^{\frac{1}{2}}$ the associated norm. For $\alpha \in \mathbb{R}^{m} \backslash\{0\}$ the reflection $\sigma_{\alpha}$ in the hyperplane orthogonal to $\alpha$ is given by

$$
\sigma_{\alpha}(x)=x-2 \frac{\langle\alpha, x\rangle}{|\alpha|^{2}} \alpha, \quad x \in \mathbb{R}^{m} .
$$

A finite set $\mathrm{R} \subset \mathbb{R}^{m} \backslash\{0\}$ is called a root system if $\alpha \mathbb{R} \cap \mathrm{R}=\{\alpha,-\alpha\}$ and $\sigma_{\alpha} \mathrm{R}=\mathrm{R}$ for all $\alpha \in \mathrm{R}$. For a given root system $\mathrm{R}$, the reflections $\sigma_{\alpha}, \alpha \in \mathrm{R}$ generate the finite group $G \subset O(m)$, called the finite reflection group (or the Coxeter group) associated with $\mathrm{R}$. 
A multiplicity function $\kappa$ on the root system $\mathrm{R}$ is a $G$-invariant function $\kappa: \mathrm{R} \rightarrow \mathbb{C}$ i.e. $\kappa(\alpha)=\kappa(h \alpha)$ for all $h \in G$. We will denote $\kappa(\alpha)$ by $\kappa_{\alpha}$.

For each fixed positive subsystem $\mathrm{R}_{+}$and multiplicity function $\kappa$ we have the Dunkl operators (also, differential-difference operators):

$$
T_{i} f(x)=\frac{\partial f(x)}{\partial x_{i}}+\sum_{\alpha \in \mathrm{R}_{+}} \kappa_{\alpha} \frac{f(x)-f\left(\sigma_{\alpha} x\right)}{\langle x, \alpha\rangle} \alpha_{i}, \quad i=1, \ldots, m,
$$

for $f \in C^{1}\left(\mathbb{R}^{m}\right)$. An important consequence is that the operators $T_{i}$ are mutually commutating, that is, $T_{i} T_{j}=T_{j} T_{i}$. The Dunkl-Laplace operator is given by

$$
\Delta_{h} f(x)=\sum_{i=1}^{m} T_{i}^{2} f(x)=\Delta f(x)+2 \sum_{\alpha \in \mathrm{R}_{+}} \kappa_{\alpha}\left(\frac{\langle\nabla f(x), \alpha\rangle}{\langle\alpha, x\rangle}-\frac{f(x)-f\left(\sigma_{\alpha} x\right)}{\langle\alpha, x\rangle^{2}}\right)
$$

with the classical Laplace operator $\triangle$ and the gradient operator $\nabla$.

We notice that $\triangle_{h}|x|^{2}=2 m+4 \gamma$, where $\gamma=\gamma_{\kappa}=\sum_{\alpha \in \mathrm{R}_{+}} \kappa(\alpha)$, and the Dunkl dimension $\mu=m+2 \gamma$. Henceforward, we assume $\kappa \geq 0$ and $\gamma_{\kappa}>0$.

We consider functions $f: \mathbb{R}^{m} \rightarrow \mathbb{R}_{0, m}$. Hereby $\mathbb{R}_{0, m}$ denotes the Clifford algebra over $\mathbb{R}^{m}$ generated by $\left\{e_{1}, e_{2}, \cdots, e_{m}\right\}$ satisfying the anti-commutation relationship $e_{i} e_{j}+e_{j} e_{i}=-2 \delta_{i j}$, where $\delta_{i j}$ is the Kronecker symbol. $\underline{x}=\sum_{i=1}^{m} x_{i} e_{i}$ is the so-called vector variable.

A Dunkl-Dirac operator in $\mathbb{R}^{m}$ for the corresponding reflection group $G$ is defined as $D_{h} f=\sum_{i=1}^{m} e_{i} T_{i} f$. Functions belonging to the kernel of the DunklDirac operator $D_{h}$ are called Dunkl-monogenic functions. Moreover, $-D_{h}^{2}=$ $\Delta_{h}$, where $\Delta_{h}$ is called the Dunkl-Laplace operator in $\mathbb{R}^{m}$. Functions belonging to the kernel of Dunkl-Laplace operator are called Dunkl-harmonic functions.

\subsection{Dunkl-Clifford analysis in $\mathbb{R}^{m \mid 2 n}$}

On a superspace of dimension $(m, 2 n)$, we have $m$ commuting (or bosonic) variables $x_{1}, \ldots, x_{m}$ and $2 n$ anti-commuting (or fermionic) variables $\grave{x}_{1}, \ldots, \grave{x}_{2 n}$ subject to

$$
x_{i} x_{j}=x_{j} x_{i}, \quad \grave{x}_{i} \grave{x}_{j}=-\grave{x}_{j} \grave{x}_{i}, \quad x_{i} \grave{x}_{j}=\grave{x}_{j} x_{i} .
$$

Furthermore we know the Clifford algebra generators $e_{1}, \ldots, e_{m}$ and the symplectic Clifford algebra generators $\grave{e}_{1}, \ldots, \grave{e}_{2 n}$. They obey the following rules:

$$
\begin{aligned}
& e_{j} e_{k}+e_{k} e_{j}=-2 \delta_{j k}, \quad \grave{e}_{2 j} \grave{e}_{2 k}-\grave{e}_{2 k} \grave{e}_{2 j}=0, \\
& \grave{e}_{2 j-1} \grave{e}_{2 k-1}-\grave{e}_{2 k-1} \grave{e}_{2 j-1}=0, \\
& \grave{e}_{2 j-1} \grave{e}_{2 k}-\grave{e}_{2 k} \grave{e}_{2 j-1}=\delta_{j k}, \quad e_{j} \grave{e}_{k}+\grave{e}_{k} e_{j}=0 .
\end{aligned}
$$

Taking the above relations into account, we study the superspace by the real algebra:

$$
A l g\left(x_{i}, e_{i} ; \grave{x}_{j}, \grave{e}_{j}\right)=\operatorname{Alg}\left(x_{i}, \grave{x}_{j}\right) \otimes \operatorname{Alg}\left(e_{i}, \grave{e}_{j}\right), i=1, \ldots, m ; j=1, \ldots, 2 n,
$$


which is nothing than the tensor product of $A l g\left(x_{i}, \grave{x}_{j}\right)$ and $A l g\left(e_{i}, \grave{e}_{j}\right)$. The algebra $\operatorname{Alg}\left(x_{i}, \grave{x}_{j}\right)$ is called a scalar algebra denoted by $\mathcal{P}$ and the algebra $\operatorname{Alg}\left(e_{i}, \grave{e}_{j}\right)$ is a Clifford algebra denoted by $\mathcal{C}_{m \mid 2 n}$. Moreover, the elements of both two algebras can commute with each other. When $n=0$, we have that $\mathcal{P} \otimes$ $\mathcal{C}_{m \mid 0}=\mathbf{R}\left[x_{1}, \ldots, x_{m}\right] \otimes \mathbf{R}_{0, m}$, with $\mathbf{R}\left[x_{1}, \ldots, x_{m}\right]$ generated by the commuting variables $x_{i}$. In the case $\mathcal{C}_{m \mid 0} \cong \mathbf{R}_{0, m}, \mathbf{R}_{0, m}$ is the standard orthogonal Clifford algebra. When $m=0$, we have that $\mathcal{P} \otimes \mathcal{C}_{0 \mid 2 n}=\Lambda_{2 n} \otimes \mathcal{W}_{2 n}$, with $\Lambda_{2 n}$ being the Grassmann algebra generated by $\grave{x}_{j}$. In the case $\mathcal{C}_{0 \mid 2 n} \cong \mathcal{W}_{2 n}, \mathcal{W}_{2 n}$ is the Weyl algebra generated by $\grave{e}_{j}$.

We define the super vector variable $x$ as $x=\underline{x}+\underline{\grave{x}}$, where $\underline{x}=\sum_{i=1}^{m} x_{i} e_{i}$ and $\underline{\grave{x}}=\sum_{j=1}^{2 n} \grave{x}_{j} \grave{e}_{j}$. By direct calculation, we obtain the square of $x$ :

$$
x^{2}=\underline{\grave{x}}^{2}+\underline{x}^{2} \text {, with } \underline{\grave{x}}^{2}=\sum_{j=1}^{n} \grave{x}_{2 j-1} \grave{x}_{2 j} \text { and } \underline{x}^{2}=-\sum_{i=1}^{m} x_{i}^{2} .
$$

Note that $\underline{x}^{2}=-\sum_{i=1}^{m} x_{i}^{2}$ is the minus norm squared of a vector in Euclidean space.

Finally, we define a more general function space as $C^{k}(\Omega) \otimes \Lambda_{2 n} \otimes \mathcal{C}_{m \mid 2 n}$, where $C^{k}(\Omega)$ denotes the space of the $k$-times continously differentiables realvalued functions defined in some domain $\Omega \subset \mathbf{R}^{m}$. We use the notation

$$
C^{k}(\Omega)_{m \mid 2 n}=C^{k}(\Omega) \otimes \Lambda_{2 n} .
$$

The super Dunkl-Dirac operator is defined to be

$$
D=-D_{h}+D_{f}=-\sum_{i=1}^{m} e_{i} T_{i}+2 \sum_{j=1}^{n}\left(\grave{e}_{2 j} \partial_{\grave{x}_{2 j-1}}-\grave{e}_{2 j-1} \partial_{\grave{x}_{2 j}}\right)
$$

with $D_{h}$ and $D_{f}$ the bosonic Dunkl-Dirac operator and the fermionic Dirac operator, respectively.

If we let $D$ act on $x$, we see that

$$
M:=\frac{1}{2} D x=-n+\frac{m}{2}+\gamma_{\kappa},
$$

where $M$ is the so-called super Dunkl dimension in contrast to the non-Dunkl case of the super-dimension $m-2 n$ in [5]. The numerical parameter $M$ is regarded as the ground level energy in physics.

As usual, functions belonging to the kernel of the super Dunkl-Dirac operator are called super Dunkl-monogenic functions.

The super Dunkl-Laplace operator is the square of the super Dunkl-Dirac operator

$$
\Delta=\Delta_{h}+\Delta_{f}=-\sum_{i=1}^{m} T_{i}^{2}+4 \sum_{j=1}^{n} \partial_{\grave{x}_{2 j-1}} \partial_{\grave{x}_{2 j}}
$$


The Dunkl-Laplace operator $\Delta_{h}$ is invariant under the Coxeter group $G$, while the fermionic Laplace operator $\Delta_{f}$ is invariant under the symplectic group.

Besides, we define the super Euler operator as

$$
\mathbf{E}=\mathbf{E}_{b}+\mathbf{E}_{f}=\sum_{i=1}^{m} x_{i} \partial_{x_{i}}+\sum_{j=1}^{2 n} \grave{x}_{j} \partial_{\grave{x}_{j}} .
$$

It is easy to decompose $\mathcal{P}$ as

$$
\mathcal{P}=\bigoplus_{k=0}^{\infty} \mathcal{P}_{k}, \quad \mathcal{P}_{k}=\{p \in \mathcal{P} \mid \mathbf{E} p=k p\}
$$

\section{Normalized system for the super Dunkl-Laplace oper- ator}

Definition 1. [2] An open connected set $\Omega \subset \mathbf{R}^{m}$ is a star domain with center 0 if any $\underline{x} \in \Omega$ and $0 \leq t \leq 1$ imply that $t \underline{x} \in \Omega$. The set is denoted by $\Omega^{\star}$.

Definition 2. Let $f(x) \in C^{1}\left(\Omega^{\star}\right)_{m \mid 2 n} \otimes \mathcal{C}_{m \mid 2 n}$. The operator $J_{s}$ is defined as

$$
J_{s} f(x)=\int_{0}^{1}(1-\alpha)^{s-1} \alpha^{M-1} f(\alpha x) d \alpha,
$$

where $s>0$ and $M>0$.

Definition 3. We define the operator $\mathbf{E}_{t}$ by

$$
\mathbf{E}_{t}=t \mathbf{I}+\mathbf{E}=t \mathbf{I}+\sum_{i=1}^{m} x_{i} \partial_{x_{i}}+\sum_{j=1}^{2 n} \grave{x}_{j} \partial_{\grave{x}_{j}},
$$

where $t \in \mathbf{R}$ and $\mathbf{I}$ is the identity operator.

Lemma 1. [24] The operators $x^{2}, \Delta, \mathbf{E}$ have the following properties:

$$
\Delta x^{2}-x^{2} \Delta=4 \mathbf{E}_{M}, \quad \mathbf{E}_{M} x^{2}-x^{2} \mathbf{E}_{M}=2 x^{2},
$$

where $M=-n+\frac{m}{2}+\gamma_{\kappa}$.

Lemma 2. Let $f(x) \in C^{2}\left(\Omega^{\star}\right)_{m \mid 2 n} \otimes \mathcal{C}_{m \mid 2 n}$. If $s \geq 1$, then

$$
\Delta\left(x^{2 s} f(x)\right)=x^{2 s} \Delta f(x)+4 s x^{2 s-2} \mathbf{E}_{M+s-1} f(x) .
$$

Proof. By Lemma 1, we have

$$
\begin{aligned}
\Delta & \left(x^{2 s} f(x)\right)=\Delta x^{2}\left(x^{2 s-2} f(x)\right)=\left(x^{2} \Delta+4 \mathbf{E}_{M}\right)\left(x^{2 s-2} f(x)\right) \\
& =x^{2} \Delta x^{2 s-2} f(x)+4 \mathbf{E}_{M} x^{2 s-2} f(x) \\
& =x^{2}\left(x^{2} \Delta+4 \mathbf{E}_{M}\right) x^{2 s-4} f(x)+4\left(x^{2} \mathbf{E}_{M}+2 x^{2}\right) x^{2 s-4} f(x) \\
& =\cdots=x^{2 s} \Delta f(x)+4 s x^{2 s-2}\left(\mathbf{E}_{M}+s-1\right) f(x) \\
& =x^{2 s} \Delta f(x)+4 s x^{2 s-2} \mathbf{E}_{M+s-1} f(x) .
\end{aligned}
$$

Thus, we have the proof. 
Lemma 3. Let $f(x) \in C^{1}\left(\Omega^{\star}\right)_{m \mid 2 n} \otimes \mathcal{C}_{m \mid 2 n}$. If $s>1$, then

$$
\mathbf{E}_{M+s-1} J_{s} f(x)=(s-1) J_{s-1} f(x) .
$$

Proof. We calculate

$$
\begin{aligned}
& \mathbf{E}_{M+s-1} J_{s} f(x)=\mathbf{E} J_{s} f(x)+(M+s-1) f(x) \\
& \quad=\left(\sum_{i=1}^{m} x_{i} \partial_{x_{i}}+\sum_{j=1}^{2 n} \grave{x}_{j} \partial_{\grave{x}_{j}}\right) \int_{0}^{1}(1-\alpha)^{s-1} \alpha^{M-1} f(\alpha x)+(M+s-1) f(x) .
\end{aligned}
$$

Let $f(\alpha x)=f_{1}(\alpha \underline{x}) f_{2}(\alpha \underline{x})$. Applying integration by parts, we have

$$
\begin{aligned}
& \int_{0}^{1}(1-\alpha)^{s-1} \alpha^{M-1} \sum_{i=1}^{m} x_{i} \partial_{x_{i}} f_{1}(\alpha \underline{x}) f_{2}(\alpha \underline{x}) d \alpha \\
& +\int_{0}^{1}(1-\alpha)^{s-1} \alpha^{M-1} \sum_{j=1}^{2 n} \grave{x}_{j} \partial_{\grave{x}_{j}} f_{1}(\alpha \underline{x}) f_{2}(\alpha \underline{\grave{x}}) d \alpha \\
& =\int_{0}^{1}(1-\alpha)^{s-1} \alpha^{M-1} \alpha \frac{\partial}{\partial \alpha} f_{1}(\alpha \underline{x}) f_{2}(\alpha \underline{\grave{x}}) d \alpha \\
& +\int_{0}^{1}(1-\alpha)^{s-1} \alpha^{M} f_{1}(\alpha \underline{x}) \alpha \frac{\partial}{\partial \alpha} f_{2}(\alpha \underline{\grave{x}}) d \alpha \\
& =\left.(1-\alpha)^{s-1} \alpha^{M} f(\alpha x)\right|_{0} ^{1} \\
& -\int_{0}^{1} f(\alpha x)\left[-(s-1)(1-\alpha)^{s-2} \alpha^{M}+M(1-\alpha)^{s-1} \alpha^{M-1}\right] d \alpha \\
& =-(M+s-1) \int_{0}^{1} f(\alpha x)(1-\alpha)^{s-1} \alpha^{M-1} d \alpha \\
& +(s-1) \int_{0}^{1} f(\alpha x)\left[(1-\alpha)^{s-2} \alpha^{M}+(1-\alpha)^{s-1} \alpha^{M-1}\right] d \alpha \\
& =-(M+s-1) \int_{0}^{1} f(\alpha x)(1-\alpha)^{s-1} \alpha^{M-1} d \alpha \\
& +(s-1) \int_{0}^{1} f(\alpha x)(1-\alpha)^{s-2} \alpha^{M-1}[\alpha+(1-\alpha)] d \alpha \\
& =-(M+s-1) J_{s} f(x)+(s-1) J_{s-1} f(x) \text {. }
\end{aligned}
$$

To sum up, we have the conclusion.

Theorem 1. Let $f(x) \in C^{2}\left(\Omega^{\star}\right)_{m \mid 2 n} \otimes \mathcal{C}_{m \mid 2 n}$. If $\Delta f(x)=0$, then the system

$$
F_{0}(x ; f)=f, \quad F_{s}(x ; f)=\frac{x^{2 s}}{4^{s} s !(s-1) !} J_{s} f, \quad(s \geq 1)
$$

is the 0-normalized system for the super Dunkl-Laplace operator, where $J_{s} f$ is given in Definition 2. 
Proof. Note that $\Delta J_{s} f=0$ for $s \geq 1$. Lemmas 2 and 3 imply that

$$
\begin{aligned}
\Delta F_{s} & =\frac{1}{4^{s} s !(s-1) !} \Delta\left(x^{2 s} f(x)\right)=\frac{x^{2(s-1)}}{4^{s-1}(s-1) !(s-1) !} \mathbf{E}_{M+s-1} J_{s} f \\
& =\frac{x^{2(s-1)}}{4^{s-1}(s-1) !(s-1) !}(s-1) J_{s-1} f=\frac{x^{2(s-1)}}{4^{s-1}(s-1) !(s-2) !} J_{s-1} f \\
& =F_{s-1} .
\end{aligned}
$$

Therefore, we obtain the conclusion.

\section{Applications of the normalized system}

4.1 Almansi type expansion for solutions of Dunkl-polyharmonic equations in superspace

Lemma 4. For $q \in \mathbf{N}$ and $l \geq 0$,

$$
(\mathbf{E}+l+1) \int_{0}^{1} \frac{(1-\alpha)^{q}}{q !} \alpha^{l} g(\alpha x) d \alpha=\int_{0}^{1} \frac{(1-\alpha)^{q-1}}{(q-1) !} \alpha^{l+1} g(\alpha x) d \alpha .
$$

For $q=0$,

$$
(\mathbf{E}+l+1) \int_{0}^{1} \alpha^{l} g(\alpha x) d \alpha=g(x)
$$

Proof. For $q=0$, we calculate

$$
\begin{aligned}
\mathbf{E} \int_{0}^{1} \alpha^{l} g(\alpha x) d \alpha & =\int_{0}^{1} \alpha^{l} \mathbf{E} g(\alpha x) d \alpha=\int_{0}^{1} \alpha^{l+1} \frac{\partial}{\partial \alpha} g(\alpha x) d \alpha \\
& =\left.\alpha^{l+1} g(\alpha x)\right|_{0} ^{1}-(l+1) \int_{0}^{1} \alpha^{l} g(\alpha x) d \alpha \\
& =g(x)-(l+1) \int_{0}^{1} \alpha^{l} g(\alpha x) d \alpha .
\end{aligned}
$$

Hence we see that

$$
(\mathbf{E}+l+1) \int_{0}^{1} \alpha^{l} g(\alpha x) d \alpha=g(x) .
$$

Then it is easy to obtain (4.1). Note that (4.1) is (4.2) for the case $q=0$. Similarly, for $q \in \mathbf{N}$ and $l \geq 0$, we have

$$
\begin{aligned}
& \mathbf{E} \int_{0}^{1} \frac{(1-\alpha)^{q}}{q !} \alpha^{l} g(\alpha x) d \alpha \\
& \quad=-(l+1) \int_{0}^{1} \frac{(1-\alpha)^{q}}{q !} \alpha^{l} g(\alpha x) d \alpha+\int_{0}^{1} \frac{(1-\alpha)^{q-1}}{(q-1) !} \alpha^{l+1} g(\alpha x) d \alpha,
\end{aligned}
$$

which completes the proof. 
Theorem 2. Let $G(x) \in C^{2 r}\left(\Omega^{*}\right)_{m \mid 2 n} \otimes \mathcal{C}_{m \mid 2 n}$. If $\Delta^{r} G(x)=0$, then

$$
G(x)=\sum_{s=0}^{r-1} F_{s}=f_{0}+\sum_{s=1}^{r-1} \frac{x^{2 s}}{4^{s} s !(s-1) !} \int_{0}^{1}(1-\alpha)^{s-1} \alpha^{M-1} f_{s}(\alpha x) d \alpha,
$$

where $\Delta f_{s}(x)=0(0 \leq s \leq r-1)$, and

$$
f_{s}(x)=\Delta^{s} G(x)+\sum_{l=1}^{r-s-1} \frac{(-1)^{l} x^{2 l}}{4^{l} l !} \int_{0}^{1} \frac{(1-\alpha)^{l-1} \alpha^{l-1}}{(l-1) !} \alpha^{M-1} \Delta^{s+l} G(\alpha x) d \alpha .
$$

Proof. First we will prove that $f_{s}(x)$ in (4.4) satisfy (4.3). Inserting (4.4) into the right-hand side of (4.3), we have

$$
\begin{aligned}
& f_{0}+\sum_{s=1}^{r-1} \frac{x^{2 s}}{4^{s} s !(s-1) !} \int_{0}^{1}(1-\alpha)^{s-1} \alpha^{M-1} f_{s}(\alpha x) d \alpha \\
= & G(x)+\sum_{s=1}^{r-1} \frac{(-1)^{s} x^{2 s}}{4^{s} s !} \int_{0}^{1} \frac{(1-\alpha)^{s-1} \alpha^{s-1}}{(s-1) !} \alpha^{M-1} \Delta^{s} G(\alpha x) d \alpha \\
& +\sum_{s=1}^{r-1} \frac{x^{2 s}}{4^{s} s !(s-1) !} \int_{0}^{1}(1-\alpha)^{s-1} \alpha^{M-1} \Delta^{s} G(\alpha x) d \alpha \\
& +\sum_{s=1}^{r-2} \frac{x^{2 s}}{4^{s} s !(s-1) !} \int_{0}^{1}(1-\alpha)^{s-1} \alpha^{M-1} \sum_{l=1}^{r-s-1} \frac{(-1)^{l}(\alpha x)^{2 l}}{4^{l} l !} \\
& \times \int_{0}^{1} \frac{(1-\beta)^{l-1} \beta^{l-1}}{(l-1) !} \beta^{\frac{M}{2}-1} \Delta^{l+s} G(\alpha \beta x) d \beta d \alpha .
\end{aligned}
$$

Denote by $A_{1}(x)$ the fourth term on the right side of the (4.5). Then

$$
\begin{aligned}
A_{1}(x)= & \sum_{s=1}^{r-2} \sum_{l=1}^{r-s-1} \frac{(-1)^{l} x^{2 s+2 l}}{4^{l+s} s ! l !} \int_{0}^{1} \frac{(1-\alpha)^{s-1} \alpha^{2 l+M-1}}{(s-1) !} \\
& \times \int_{0}^{1} \frac{(1-\beta)^{l-1} \beta^{l+M-2}}{(l-1) !} \Delta^{l+s} G(\alpha \beta x) d \beta d \alpha=\sum_{s=1}^{r-2} \sum_{l=1}^{r-s-1} \frac{(-1)^{l} x^{2 s+2 l}}{4^{l+s} s ! l !} \\
& \times \int_{0}^{1} \frac{\alpha^{2}(1-\alpha)^{s-1}}{(s-1) !} \int_{0}^{1} \frac{(\alpha-\alpha \beta)^{l-1}(\alpha \beta)^{l+M-2}}{(l-1) !} \Delta^{l+s} G(\alpha \beta x) d \beta d \alpha .
\end{aligned}
$$

Denote by $A_{2}(x)$ the integral in the above expression. Let $t=\alpha \beta$, then $d t=$ $\alpha d \beta$ for $0 \leq \alpha \leq 1$ and $0 \leq t \leq \alpha$. We calculate

$$
\begin{aligned}
A_{2}(x) & =\int_{0}^{1} \frac{\alpha(1-\alpha)^{s-1}}{(s-1) !} \int_{0}^{\alpha} \frac{(\alpha-t)^{l-1} t^{l+M-2}}{(l-1) !} \Delta^{l+s} G(t x) d t d \alpha \\
& =\int_{0}^{1} \int_{0}^{\alpha} \frac{\alpha(1-\alpha)^{s-1}(\alpha-t)^{l-1}}{(s-1) !(l-1) !} t^{l+M-2} \Delta^{l+s} G(t x) d t d \alpha \\
& =\int_{0}^{1} \int_{t}^{1} \frac{\alpha(1-\alpha)^{s-1}(\alpha-t)^{l-1}}{(s-1) !(l-1) !} t^{l+M-2} \Delta^{l+s} G(t x) d \alpha d t
\end{aligned}
$$




$$
=\int_{0}^{1} \frac{t^{l+M-2}}{(s-1) !(l-1) !} \Delta^{l+s} G(t x) d t \int_{t}^{1} \alpha(1-\alpha)^{s-1}(\alpha-t)^{l-1} d \alpha .
$$

Let $\alpha=\beta+t$. Then $d \beta=d \alpha$. For $0 \leq \beta \leq 1-t$,

$$
A_{3}(t)=\int_{t}^{1} \alpha(1-\alpha)^{s-1}(\alpha-t)^{l-1} d \alpha=\int_{0}^{1-t}(\beta+t)(1-\beta-t)^{s-1} \beta^{l-1} d \beta .
$$

Let $\beta=\alpha(1-t)$. Then $d \beta=(1-t) d \alpha$ for $0 \leq \alpha \leq 1$. We see that

$$
\begin{aligned}
A_{3}(t) & =\int_{0}^{1}(\alpha-\alpha t+t)(1-\alpha)^{s-1}(1-t)^{s-1} \alpha^{l-1}(1-t)^{l} d \alpha \\
& =(1-t)^{s+l-1} \int_{0}^{1}(\alpha-\alpha t+t)(1-\alpha)^{s-1} \alpha^{l-1} d \alpha .
\end{aligned}
$$

We calculate

$$
\begin{aligned}
A_{3}(t) & =(1-t)^{l+s} \int_{0}^{1} \alpha^{l}(1-\alpha)^{s-1} d \alpha+t(1-t)^{l+s-1} \int_{0}^{1} \alpha^{l-1}(1-\alpha)^{s-1} d \alpha \\
& =(1-t)^{l+s} B(l+1, s)+t(1-t)^{s+l-1} B(l, s),
\end{aligned}
$$

where

$$
B(l, s)=\int_{0}^{1} \alpha^{l-1}(1-\alpha)^{s-1} d \alpha .
$$

Using the relation between Beta functions and Gamma functions:

$$
B(l, s)=\frac{\Gamma(l) \Gamma(s)}{\Gamma(s+l)}, \quad \Gamma(s)=(s-1) !
$$

we can write

$$
\begin{aligned}
A_{3}(t) & =\frac{\Gamma(l+1) \Gamma(s)}{\Gamma(l+s+1)}(1-t)^{s+l}+\frac{\Gamma(l) \Gamma(s)}{\Gamma(l+s)} t(1-t)^{l+s-1} \\
& =\frac{l !(s-1) !}{(l+s) !}(1-t)^{s+l}+\frac{(l-1) !(s-1) !}{(l+s-1) !} t(1-t)^{l+s-1} .
\end{aligned}
$$

Inserting $A_{3}(t)$ into $A_{1}(x)$, we have

$$
\begin{aligned}
& \sum_{s=1}^{r-2} \sum_{l=1}^{r-s-1} \frac{(-1)^{l} x^{2 s+2 l}}{4^{l+s}} \int_{0}^{1} \frac{(1-t)^{s+l}}{s !(l-1) !(l+s) !} t^{l+M-2} \Delta^{l+s} G(t x) d t \\
& +\sum_{s=1}^{r-2} \sum_{l=1}^{r-s-1} \frac{(-1)^{l} x^{2 s+2 l}}{4^{l+s}} \int_{0}^{1} \frac{t(1-t)^{l+s-1}}{s ! l !(l+s-1) !} t^{l+M-2} \Delta^{l+s} G(t x) d t \\
= & \sum_{s=1}^{r-2} \sum_{l=1}^{r-s-1} \frac{(-1)^{l} x^{2 s+2 l}}{4^{l+s}} \int_{0}^{1} \frac{t^{l-1}(1-t)^{s+l}}{s !(l-1) !(l+s) !} t^{M-1} \Delta^{l+s} G(t x) d t \\
& +\sum_{s=1}^{r-2} \sum_{l=1}^{r-s-1} \frac{(-1)^{l} x^{2 s+2 l}}{4^{l+s}} \int_{0}^{1} \frac{t^{l}(1-t)^{l+s-1}}{s ! l !(l+s-1) !} t^{M-1} \Delta^{l+s} G(t x) d t
\end{aligned}
$$




$$
\begin{aligned}
= & \sum_{i=2}^{r-1} \sum_{l=1}^{i-1} \frac{(-1)^{l} x^{2 i}}{4^{i}} \int_{0}^{1}\left[\frac{t^{i-l-1}(1-t)^{i}}{l !(i-l-1) ! i !}+\frac{t^{i-l}(1-t)^{i-1}}{l !(i-l) !(i-1) !}\right] t^{M-1} \Delta^{i} G(t x) d t \\
= & \sum_{i=2}^{r-1} \frac{x^{2 i}}{4^{i}} \int_{0}^{1}\left[\frac{(1-t)^{i}}{i !} \sum_{l=1}^{i-1} \frac{(-1)^{l} t^{i-l-1}}{l !(i-l-1) !}\right] t^{M-1} \Delta^{i} G(t x) d t \\
& +\sum_{i=2}^{r-1} \frac{x^{2 i}}{4^{i}} \int_{0}^{1}\left[\frac{(1-t)^{i-1}}{(i-1) !} \sum_{l=1}^{i-1} \frac{(-1)^{l} t^{i-l}}{l !(i-l) !}\right] t^{M-1} \Delta^{i} G(t x) d t .
\end{aligned}
$$

We calculate

$$
\sum_{l=1}^{i-1} \frac{(-1)^{l} t^{i-l-1}}{l !(i-l-1) !}=\sum_{l=0}^{i-1} \frac{(-1)^{l} t^{i-l-1}}{l !(i-l-1) !}-\frac{t^{i-1}}{(i-1) !}=\frac{(t-1)^{i-1}}{(i-1) !}-\frac{t^{i-1}}{(i-1) !}
$$

and

$$
\sum_{l=1}^{i-1} \frac{(-1)^{l} t^{i-l}}{l !(i-l) !}=\sum_{l=0}^{i-1} \frac{(-1)^{l} t^{i-l}}{l !(i-l) !}-\frac{t^{i}}{i !}-\frac{(-1)^{i}}{i !}=\frac{(t-1)^{i}}{i !}-\frac{t^{i}}{i !}-\frac{(-1)^{i}}{i !} .
$$

Thus, we have

$$
\begin{array}{r}
A_{1}(x)=\sum_{i=2}^{r-1} \frac{x^{2 i}}{4^{i}} \int_{0}^{1}\left[-\frac{(1-t)^{i-1} t^{i-1}}{(i-1) ! i !}-\frac{(-1)^{i}(1-t)^{i-1}}{(i-1) ! i !}\right] t^{M-1} \Delta^{i} G(t x) d t \\
=\sum_{i=1}^{r-1} \frac{x^{2 i}}{4^{i}} \int_{0}^{1}\left[-\frac{(1-t)^{i-1} t^{i-1}}{(i-1) ! i !}-\frac{(-1)^{i}(1-t)^{i-1}}{(i-1) ! i !}\right] t^{M-1} \Delta^{i} G(t x) d t .
\end{array}
$$

Inserting $A_{1}(x)$ into (4.5), we have (4.3).

Next, we will prove that $\Delta f_{s}(x)=0$. By Lemma 2, we have

$$
\begin{aligned}
& \Delta f_{s}(x) \\
= & \Delta^{s+1} G(x)+\sum_{l=1}^{r-s-1} \frac{(-1)^{l}}{4^{l} l !} \Delta\left(x^{2 l} \int_{0}^{1} \frac{(1-\alpha)^{l-1} \alpha^{l-1}}{(l-1) !} \alpha^{M-1} \Delta^{s+l} G(\alpha x) d \alpha\right) \\
= & \Delta^{s+1} G(x)+\sum_{l=1}^{r-s-2} \frac{(-1)^{l} x^{2 l}}{4^{l} l !} \int_{0}^{1} \frac{(1-\alpha)^{l-1} \alpha^{l+1}}{(l-1) !} \alpha^{M-1} \Delta^{s+l+1} G(\alpha x) d \alpha \\
& -\sum_{l=1}^{r-s-1} \frac{(-1)^{l} x^{2(l-1)}}{4^{l-1}(l-1) !}(\mathbf{E}+M+l-1) \int_{0}^{1} \frac{(1-\alpha)^{l-1} \alpha^{l-1}}{(l-1) !} \alpha^{M-1} \Delta^{s+l} G(\alpha x) d \alpha .
\end{aligned}
$$

By means of Lemma 4, we have

$$
\begin{aligned}
& \sum_{l=1}^{r-s-1} \frac{(-1)^{l} x^{2(l-1)}}{4^{l-1}(l-1) !}(\mathbf{E}+M+l-1) \int_{0}^{1} \frac{(1-\alpha)^{l-1} \alpha^{l-1}}{(l-1) !} \alpha^{M-1} \Delta^{s+l} G(\alpha x) d \alpha \\
& \quad=-(\mathbf{E}+M) \int_{0}^{1} \alpha^{M-1} \Delta^{s+1} G(\alpha x) d \alpha-\sum_{l=2}^{r-s-1} \frac{(-1)^{l-1} x^{2(l-1)}}{4^{l-1}(l-1) !}
\end{aligned}
$$




$$
\begin{aligned}
& \times(\mathbf{E}+M+l-1) \int_{0}^{1} \frac{(1-\alpha)^{l-1} \alpha^{l-1}}{(l-1) !} \alpha^{M-1} \Delta^{s+l} G(\alpha x) d \alpha \\
&=-\Delta^{s+1} G(x)-\sum_{l=2}^{r-s-1} \frac{(-1)^{l-1} x^{2(l-1)}}{4^{l-1}(l-1) !} \int_{0}^{1} \frac{(1-\alpha)^{l-2} \alpha^{l}}{(l-2) !} \alpha^{M-1} \Delta^{s+l} G(\alpha x) d \alpha \\
&=-\Delta^{s+1} G(x)-\sum_{l=2}^{r-s-1} \frac{(-1)^{l-1} x^{2(l-1)}}{4^{l-1}(l-1) !} \int_{0}^{1} \frac{(1-\alpha)^{l-2} \alpha^{l}}{(l-2) !} \alpha^{M-1} \Delta^{s+l} G(\alpha x) d \alpha \\
&=-\Delta^{s+1} G(x)-\sum_{l=1}^{r-s-2} \frac{(-1)^{l} x^{2 l}}{4^{l} l !} \int_{0}^{1} \frac{(1-\alpha)^{l-1} \alpha^{l+1}}{(l-1) !} \alpha^{M-1} \Delta^{s+l+1} G(\alpha x) d \alpha .
\end{aligned}
$$

As suggested above, we get

$$
\Delta f_{s}(x)=0
$$

Therefore, we have the conclusion.

\subsection{Solutions of the Dunkl-Helmholtz equation in superspace}

In this subsection, we investigate the solutions of the Dunkl-Helmholz equation in superspace

$$
(\Delta+\lambda) G(x)=0,
$$

where the constant $\lambda \in \mathbf{C}, G(x) \in C^{1}\left(\Omega^{*}\right)_{m \mid 2 n} \otimes \mathcal{C}_{m \mid 2 n}$.

Theorem 3. Let $f(x) \in C^{\infty}\left(\Omega^{*}\right)_{m \mid 2 n} \otimes \mathcal{C}_{m \mid 2 n}$. Suppose that the function $f(x)$ is super Dunkl-harmonic and $M>0$. Then

$$
G(x)=\sum_{s=0}^{\infty}(-\lambda)^{s} F_{s}(x ; f)
$$

is a formal solution for the equation (4.6), where $F_{s}(x ; f)$ are given in Theorem 1.

Proof. By Theorem 1, we have

$$
\begin{aligned}
& (\Delta+\lambda) G(x)=(\Delta+\lambda) F_{0}(x ; f)+(\Delta+\lambda) \lambda F_{1}(x ; f)+(\Delta+\lambda) \lambda^{2} F_{2}(x ; f)+\cdots \\
& \quad=-\lambda F_{0}(x ; f)+\lambda F_{0}(x ; f)-\lambda^{2} F_{1}(x ; f)+\lambda^{2} F_{1}(x ; f)-\cdots=0 .
\end{aligned}
$$

So the series $G(x)$ satisfies formally equation (4.6).

\subsection{Solutions of the Dunkl-Poisson equation and the inhomoge- neous Dunkl-polyharmonic equation in superspace}

Consider the Dunkl-Poisson equation in superspace

$$
\Delta g=f(x)
$$

where $f(x) \in C^{\infty}\left(\Omega^{*}\right)_{m \mid 2 n} \otimes \mathcal{C}_{m \mid 2 n}$. 
Theorem 4. Let $f(x) \in C^{\infty}\left(\Omega^{*}\right)_{m \mid 2 n} \otimes \mathcal{C}_{m \mid 2 n}$. Then the function $G(x)$ given by

$$
G(x)=\sum_{s=0}^{\infty} \frac{(-1)^{s} x^{2(s+1)}}{4^{s+1}(s+1) ! s !} \int_{0}^{1}(1-\alpha)^{s} \alpha^{M+s-1} \Delta^{s} f(\alpha x) d \alpha
$$

is a formal solution of the equation (4.8).

Proof. By Lemmas 2 and 4, we have

$$
\begin{aligned}
& \Delta G(x)=\sum_{s=0}^{\infty} \frac{(-1)^{s}}{4^{s+1}(s+1) ! s !} \Delta\left(x^{2(s+1)} \int_{0}^{1}(1-\alpha)^{s} \alpha^{M+s-1} \Delta^{s} f(\alpha x) d \alpha\right) \\
& =\sum_{s=0}^{\infty} \frac{(-1)^{s}}{4^{s+1}(s+1) ! s !}\left(x^{2(s+1)} \int_{0}^{1}(1-\alpha)^{s} \alpha^{M+s+1} \Delta^{s+1} f(\alpha x) d \alpha\right) \\
& +\sum_{s=0}^{\infty} \frac{(-1)^{s} x^{2 s}}{4^{s} s ! s !} \mathbf{E}_{M+s} \int_{0}^{1}(1-\alpha)^{s} \alpha^{M+s-1} \Delta^{s} f(\alpha x) d \alpha \\
& =\sum_{s=0}^{\infty} \frac{(-1)^{s}}{4^{s+1}(s+1) ! s !}\left(x^{2(s+1)} \int_{0}^{1}(1-\alpha)^{s} \alpha^{M+s+1} \Delta^{s+1} f(\alpha x) d \alpha\right) \\
& +\mathbf{E}_{M} \int_{0}^{1} \alpha^{M-1} f(\alpha x) d \alpha \\
& +\sum_{s=1}^{\infty} \frac{(-1)^{s} x^{2 s}}{4^{s} s ! s !} \mathbf{E}_{M+s} \int_{0}^{1}(1-\alpha)^{s} \alpha^{M+s-1} \Delta^{s} f(\alpha x) d \alpha \\
& =\sum_{s=0}^{\infty} \frac{(-1)^{s}}{4^{s+1}(s+1) ! s !}\left(x^{2(s+1)} \int_{0}^{1}(1-\alpha)^{s} \alpha^{M+s+1} \Delta^{s+1} f(\alpha x) d \alpha\right) \\
& +(\mathbf{E}+M) \int_{0}^{1} \alpha^{M-1} f(\alpha x) d \alpha \\
& +\sum_{s=1}^{\infty} \frac{(-1)^{s} x^{2 s}}{4^{s} s !} \int_{0}^{1} \frac{(1-\alpha)^{s-1}}{(s-1) !} \alpha^{M+s} \Delta^{s} f(\alpha x) d \alpha \\
& =\sum_{s=0}^{\infty} \frac{(-1)^{s} x^{2(s+1)}}{4^{s+1}(s+1) ! s !} \int_{0}^{1}(1-\alpha)^{s} \alpha^{M+s+1} \Delta^{s+1} f(\alpha x) d \alpha+f(x) \\
& -\sum_{s=0}^{\infty} \frac{(-1)^{s}}{4^{s+1}(s+1) ! s !}\left(x^{2(s+1)} \int_{0}^{1}(1-\alpha)^{s} \alpha^{M+s+1} \Delta^{s+1} f(\alpha x) d \alpha\right) \\
& =f(x) \text {. }
\end{aligned}
$$

Thus, we complete the proof.

Consider the inhomogeneous Dunkl-polyharmonic equation in superspace

$$
\Delta^{k} g=f(x)
$$

where $f(x) \in C^{\infty}\left(\Omega^{*}\right)_{m \mid 2 n} \otimes \mathcal{C}_{m \mid 2 n}$.

Applying Theorem 4, we can obtain the following theorem by induction. 
Theorem 5. Let $f(x) \in C^{\infty}\left(\Omega^{*}\right)_{m \mid 2 n} \otimes \mathcal{C}_{m \mid 2 n}$. Then the function $G(x)$ given by

$$
G(x)=\frac{x^{2 k}}{2(2 k-2) ! !} \sum_{s=0}^{\infty} \frac{(-1)^{s} x^{2 s}}{(2 s+2 k) ! !(2 s) ! !} \int_{0}^{1}(1-\alpha)^{s+k-1} \alpha^{M+s-1} \Delta^{s} f(\alpha x) d \alpha
$$

is a formal solution of the equation (4.10).

Remark 1. If the series $G(x)$ in (4.7) converges absolutely and uniformly, then it is a classical solution of the equation (4.6). Similarly, the series $G(x)$ in (4.9) and (4.11) can be considered.

\section{Acknowledgements}

This research was supported by the TianYuan Special Funds of the National Natural Science Foundation of China (No. 11426082).

\section{References}

[1] E. Almansi. Sull'integrazione dell'equazione differenziale $\triangle^{2 m} u=0$. Annali di Mat. Pura ed Appl., 2(1):1-51, 1899. http://dx.doi.org/10.1007/BF02419286.

[2] N. Aronszajn, T.M. Creese and L.J. Lipkin. Polyharmonic functions. Oxford Mathematics Monographs, The Clarendon Press, Oxford University Press, New York, 1983.

[3] G. Bernardes, P. Cerejeiras and U. Kähler. Fischer decomposition and Cauchy kernel for Dunkl-Dirac operators. Adv. Appl. Clifford Alg., 19:163-171, 2009.

[4] H. De Bie and F. Sommen. Correct rules for Clifford calculus on superspace. Adv. Appl. Clifford Alg., 17(3):357-382, 2007. http://dx.doi.org/10.1007/s00006-007-0042-y.

[5] H. De Bie and F. Sommen. Spherical harmonics and integration in superspace. J. Phys. A: Math. Theor., 40(26):7193-7212, 2007. http://dx.doi.org/10.1088/1751-8113/40/26/007.

[6] B.A. Bondarenko. Operatornye algoritmy v Differenzialnyh Uravneniyah. Fan, Tashkent, 1984.

[7] F. Brack, R. Delanghe and F. Sommen. Clifford analysis. Res Notes Math, Pitman, London, 1982.

[8] P. Cerejeiras, U. Kähler and G. B. Ren. Clifford analysis for finite reflection groups. Adv. Appl. Clifford Alg., 51:487-495, 2006.

[9] Ch. F. Dunkl. Differential-difference operators associated to reflection groups. Trans. Amer. Math. Soc., 311:167-183, 1989. http://dx.doi.org/10.1090/S0002-9947-1989-0951883-8.

[10] Ch. F. Dunkl and Y. Xu. Orthogonal polynomials of several variables. Cambridge Univ. Press, Cambridge, UK, 2001. http://dx.doi.org/10.1017/CBO9780511565717.

[11] M.G. Fei, P. Cerejeiras and U. Kähler. Fueter's theorem and its generalizations in Dunkl-Clifford analysis. J. Phys. A: Math. and Theor., 42(39):395209, 2009. http://dx.doi.org/10.1088/1751-8113/42/39/395209. 
[12] M.G. Fei, P. Cerejeiras and U. Kähler. Spherical Dunkl-monogenics and a factorization of the Dunkl-Laplacian. J. Phys. A: Math. Theor., 43(44):445202, 2010. http://dx.doi.org/10.1088/1751-8113/43/44/445202.

[13] G. J. Heckman. Dunkl operators. Astérisque, 245:223-246, 1997.

[14] V.V. Karachik. Polynomial solutions to the systems of partial differential equations with constant coefficients. Yokohama Math J., 47:121-142, 2000.

[15] V.V. Karachik. Normalized system of functions with respect to the Laplace operator and its applications. J. Math. Anal. Appl., 287(2):577-592, 2003. http://dx.doi.org/10.1016/S0022-247X(03)00583-3.

[16] V.V. Karachik. On one representation of analytic functions by harmonic functions. Mat. Tr., 10(2):142-162, 2007.

[17] V.V. Karachik. Construction of polynomial solutions to some boundary value problems for Poisson's equation. Computational Mathematics and Mathematical Physics, 51(9):1567-1587, 2011. http://dx.doi.org/10.1134/S0965542511090120.

[18] V.V. Karachik. On solvability conditions for the Neumann problem for a polyharmonic equation in the unit ball. Journal of Applied and Industrial Mathematics, 8(1):63-75, 2014. http://dx.doi.org/10.1134/S1990478914010074.

[19] L. Liu. Almansi type decompositions and their aplications. Phd. dissertation, University of Science and Technology of China, 2009.

[20] L. Liu and G. B. Ren. Normalized system for wave and Dunkl operators. Taiwanese J. Math, 14(2):675-683, 2007.

[21] H. R. Malonek and G. B. Ren. Almansi-type theorems in Clifford analysis. Math. Meth. Appl. Sci., 25:1541-1552, 2002. http://dx.doi.org/10.1002/mma.387.

[22] Y.Y. Qiao, H.F. Yuan and H.J. Yang. Normalized system for the super Laplace operator. Adv. Appl. Clifford Alg., 22(4):1109-1128, 2012.

[23] G. B. Ren. Almansi decomposition for Dunkl operators. Science in China Series A: Mathematics, 48(1):333-342, 2005. http://dx.doi.org/10.1007/BF02884718.

[24] G. B. Ren. Howe duality in Dunkl superspace. Science China Mathematics, 53(12):3153-3169, 2010. http://dx.doi.org/10.1007/s11425-010-4063-y.

[25] J. Ryan. Iterated Dirac operators in $c^{n}$. Zeitschrift für Analysis und ihre Anwendungen, 9(5):385-401, 1990.

[26] J. F. van Diejen and L. Vinet. Calogero-Moser-Sutherland Models. SpringerVerlag, New York, 2000. http://dx.doi.org/10.1007/978-1-4612-1206-5.

[27] H.F. Yuan and Y.Y. Qiao. Solutions of the Dirac and related equations in superspace. Complex Variables and Elliptic Equations: An International Journal, 59(9):1315-1327, 2014. http://dx.doi.org/10.1080/17476933.2013.836187.

[28] H.F. Yuan, Y.Y. Qiao and H.J. Yang. Decomposition of k-monogenic functions in superspace. Complex Variables and Elliptic Equations: An International Journal, 58(8):1109-1124, 2013. http://dx.doi.org/10.1080/17476933.2011.636149. 